Methods 32 patients with SSS or AVB associated chronic heart dysfunction were divided two groups RA-URIS pacing group (13 cases) and RA-RVA pacing group (19 cases). The parameters including left ventricular mass index (LVMI), left ventricular ejection fraction (LVEF) and 6 min walk test (6-MWT) were compared between two groups in pre-pacing and in follow up 24 months after pacing.

Results There were no difference on LVEF, LVMI and 6-MWT between two groups before pacemaker implanted. But after 24 months for pacing therapy in RA-URIS group, there was significant increase in LVEF (48.3 \pm 10.1 vs $40.7 \pm 8.4, \mathrm{p}<0.05)$, 6MWT $(386 \pm 69$ vs $330 \pm 78, \mathrm{p}<0.05)$ and decrease in LVMI $(102.5 \pm 16.3 \mathrm{vs}$ $120.1 \pm 18.5, \mathrm{p}<0.05)$ Meanwhile, LVEF, 6-MWT (48.3 \pm 10.1 vs $43.7 \pm 5.5,386 \pm 69$ vs $329 \pm 91, \mathrm{p}<0.05)$ were increased and LVMI $(102.5 \pm 16.3$ vs $113.6 \pm 17.4, p<0.05)$ were decreased significantly in RA-URIS group compared with those of RA-RVA group in 24 months follow up.

Conclusion The results showed that RA-URIS pacing may reverse left ventricular remodelling in patients with chronic heart dysfunction as well as improving life quality.

\section{e0650 THE EFFECTS OF BIVENTRICULAR SYNCHRONOUS PACING ON CBF, MVO2 AND CWE IN DOGS}

doi:10.1136/hrt.2010.208967.650

Fu Xianghua, Ma Ning, Li Shiqiang, Wu Weili, Li Liang, Gu Xinshun, Jiang Yunfa. The 2nd Hospital of Hebei Medical University, Shijiazhuang, Hebei, China

Objective To compare the effects of biventricular synchronous pacing with different pacing site on coronary Blood flow (CBF), myocardial oxygen consumption $\left(\mathrm{MVO}_{2}\right)$ and cardiac work efficiency (CWE).

Methods RA-cHisB and RA-RVA sequential pacing, RVA-LVPL and cHisB-LVPL pacing, RA-RVA-LVPL and RA-cHisB-LVPL pacing were randomly performed in 14 dogs with general-anaesthetised, opened chest and artificial-ventilation. SNR was as self-control. Every pacing mode was to capture SNR for 20 min with a recovery of physiologic parameters for $10 \mathrm{~min}$, and then shift another pacing mode in turn. $\mathrm{CFB}$ and $\mathrm{CO}$ were measured by a electromagnetic flowmeter. Blood sample were respectively collected from the catheters in left ventricle and coronary sinus for getting the arterial $\mathrm{O}_{2}$ saturation $\left(\mathrm{SaO}_{2}\right)$, coronary sinus $\mathrm{O}_{2}$ saturation $\left(\mathrm{Scs}_{2}\right)$ and $\mathrm{Hgb}$.

Results No significant difference in CBF among the RA-cHisB-LVPL, RA-RVA-LVPL and RA-cHisB pacing were found. CBF in RA-RVA pacing was decreased than that in RA-cHisB-LVPL, RA-RVA-LVPL and RA-cHisB pacing. $\mathrm{MVO}_{2}$ among the all groups had no significant changes compared with each other. CO of RA-cHisB, RVALVPL and CHisB-LVPL pacing were increased as compared with that in RA-RVA pacing $(p<0.01)$. CO in cHisB-LVPL pacing was increased by $6.7 \%$ than that in RVA-LVPL pacing. CO in RA-cHisB pacing was increased by $8.7 \%$ than that in RA-RVA pacing. CO in RA-RVA-LVPL pacing was increased as compared with that in RAcHisB and cHisB-LVPL pacing, respectively. CO in RA-cHisB-LVPL pacing was increased than that in RA-RVA-LVPL pacing. The changes of CWE were similar to that of $\mathrm{CO}$ among all pacing groups. CWE in RA-cHisB-LVPL pacing was significantly enhanced as compared with that in RA-cHisB and RA-RVA-LVPL pacing. Conclusions RA-cHisB dual chamber, cHisB-LVPL biventricular and RA-cHisB-LVPV triple-chamber pacing might significantly increase $\mathrm{CBF}$ and CWE without the increment of $\mathrm{MVO}_{2}$.

Conclusion The biventricular synchronous pacing have the beneficial effects on maintaining the balance between myocardial oxygen supply and consumption and increasing CWE by enhancing the cardiac ejection performance.

\section{E0651 EVALUATION ON ACUTE HAEMODYNAMIC EFFECTS OF INTRAVENOUS RHBNP IN ACUTE MYOCARDIAL INFARCTION PATIENTS WITH HEART FAILURE BY CONTINUOUS SWAN-GANZ CATHETER MONITORING}

doi:10.1136/hrt.2010.208967.651

Fu Xianghua, Li Shiqiang, Fan Weize, Gu Xinshun, Liu Jun, Hao Guozhen, Jiang Yunfa, Ma Ning, Zhang Jing, Fan Xinna. The 2nd Hospital of Hebei Medical University, Shijiazhuang, Hebei, China

Objectives To compared and evaluated the acute haemodynamic effects and safety of intravenous rhBNP versus nitroglycerin (NIT) in AMI patients with ADHF by Swan-Ganz catheter (6F, ARROW, Inc USA) monitoring through a prospectively designed study.

Methods 42 consecutive AMI patients with ADHF were randomised into rhBNP group $\left(\mathrm{n}=21,1.5 \mu \mathrm{g} \cdot \mathrm{kg}^{-1}\right.$ bolus intravenous injection followed by $0.0075 \mu \mathrm{g} \cdot \mathrm{kg}^{-1} \cdot \mathrm{min}^{-1}$ for the first $3 \mathrm{~h}$ and $0.015-0.03 \mu \mathrm{g} \cdot \mathrm{kg}^{-1} \cdot \mathrm{min}^{-1}$ infusion for following $21 \mathrm{~h}$ ) and NIT group ( $\mathrm{n}=21,10$ to $100 \mu \mathrm{g} \cdot \mathrm{min}^{-1}$ intravenous infusion for $24 \mathrm{~h}$ ) The invasive haemodynamic parameters were measured at the baseline, during $24 \mathrm{~h}$ of drug infusion and $6 \mathrm{~h}$ of post-infusion by Swan-Ganz catheter monitoring via subclavian access while total urine output during $30 \mathrm{~h}$ and relative serum chemistries were measured. MACE was followed up 1 week.

Results As early as 30 min after the initiation of rhBNP, PCWP was reduced by $48.9 \%$ contrasted to baseline and cardiac index (CI) was increased by $27.1 \%$ at $1 \mathrm{~h}$ of rhBNP infusion respectively, $(\mathrm{p}<0.05)$; these significant changes in PCWP and CI continued throughout $24 \mathrm{~h}$ of rhBNP infusion and $6 \mathrm{~h}$ of discontinuing the infusion $(p<0.05)$. Although PCWP reduced significantly at $2 \mathrm{~h}$ of NIT infusion $(p<0.05)$ and $C I$ elevated significantly at $3 \mathrm{~h}$ of infusion. The total urine output for $30 \mathrm{~h}$ of this study in rhBNP group tended to be more than that in NIT group ( $p>0.05$ ), while serum potassium concentration in rhBNP group was significantly increased relative to baseline value $\left(3.4 \pm 0.5\right.$ vs $\left.4.0 \pm 0.4 \mathrm{mmol} \cdot \mathrm{L}^{-1}, \mathrm{p}<0.05\right)$. There was no symptomatic hypotension or other adverse events appeared to be associated with rhBNP administration under this study.

Conclusions Intravenous injection of rhBNP results in more rapid, strong and prolong haemodynamic improvement than that of NIT in AMI patients with ADHF as well it is also feasible and safe in clinic as a selective agent for AMI patients with ADHF.

\section{e0652 THE LEVEL CHANGES OF IL-8 AND THE RELATIONSHIP TO THE LEFT VENTRICULAR ANEURYSIM FORMATION AND CARDIAC PERFORMANCE IN PATIENTS WITH ACUTE MYOCARDIAL INFARCTION}

doi:10.1136/hrt.2010.208967.652

Fu Xianghua, Liu Jun, Wu Weili, Wang Xuechao, Wang Yanbo, Fan Weize. The 2nd Hospital of Hebei Medical University, Shijiazhuang, Hebei, China

Objective To investigate the changes in interleukin-8 (IL-8) and the relationship with the left ventricular aneurysm (LVA) and cardiac function in acute myocardial infarction (AMI) patients using left ventriculography (LVG).

Methods A total of 106 patients with primary anterior AMI accompanied LVA diagnosed by LVG were submitted to LVG after onset of AMI symptom and divided into LVA group and non-LVA group. Plasma IL-8 was measured. At the immediately after PCI and 6th month after AMI, the parameters of left ventricular end diastolic volume index (LVEDVI), left ventricular end systolic volume index (LVESVI), left ventricular ejection fraction (LVEF), wall motion score (WMS) and left ventricular end diastolic pressure (LVEDP) were measured by LVG. The main adverse cardiac events were recorded in 24 th week after PCI. 